\title{
Fonoterapia para mulheres transgênero
}

\author{
Speech therapy for transgender women \\ Terapia del habla para mujeres transgénero
}

\section{Resumo}

Objetivo: abordar aspectos relacionados à disforia de gênero e à terapia fonoaudiológica, organizando e fornecendo informações sobre os princípios terapêuticos. Métodos: trata-se de uma revisão de literatura por meio de pesquisa em artigos científicos nas bases de dados Pubmed e Science Direct sobre o tema disforia de gênero e voz, nos últimos 20 anos. A pesquisa foi realizada, pelos autores do estudo, de janeiro de 2018 a setembro de 2021, com as palavraschave: (Pessoas trans) OR (Transexualismo) OR (Transexual) OR (Identidade de Gênero)) AND ((treinamento vocal) OR (voz) OR (qualidade de voz)). Resultados: 31 artigos científicos foram incluídos no estudo. Conclusão: há necessidade de acompanhamento multiprofissional e ratifica-se a importância da fonoterapia, uma vez que o tratamento hormonal e as cirurgias laríngeas não contemplam todas as alterações desejadas na voz e na fala em mulheres transgênero. Dessa forma, o fonoaudiólogo, além de buscar a elevação da f0, deve estimular os maneirismos femininos, minúcias da voz e delicadezas da fala. Verificou-se a importância da realização de cirurgia laríngea associada à fonoterapia para modificação vocal de mulheres transgênero.

Palavras-chave: Terapia vocal; Transgênero; Gênero e voz; Voz.

\begin{abstract}
Objective: to address aspects related to gender dysphoria and speech therapy, by organizing and providing information about therapeutic principles. Methods: this is a literature review through research in scientific articles in the database Pubmed and Science Direct on the theme of gender dysphoria and voice, in the last 20 years. The research was conduct, by the study authors, from January 2018 to September 2021, with the keywords: (Transgender Persons) OR (Transsexualism) OR (Transsexual) OR (Gender Identity)) AND ((voice training) OR (voice) OR (voice quality)). Results: 31 scientific articles were included in the study. Conclusion: there is a need for multi-professional follow-up and ratifies the importance of speech therapy, since hormonal treatment and laryngeal surgeries do not include all the desired changes in voice and speech in transsexual women. In this way, the speech therapist, besides seeking the elevation of f0, should stimulate the feminine mannerisms, minutiae of the voice and delicacies of the speech. The importance of performing laryngeal surgery combined with speech therapy for voice modification of transgender women was verify.
\end{abstract}

Keywords: Voice therapy; Transgender; Gender and voice; Voice.

\section{Resumen}

Objetivo: abordar aspectos relacionados con la disforia de género y logopedia, organizando y proporcionando información sobre principios terapéuticos. Métodos: se trata de una revisión de la literatura a través de la investigación en artículos científicos en las bases de datos Pubmed y Science Direct sobre el tema de la disforia de 
género y la voz, en los últimos 20 años. La investigación fue realizada por los autores del estudio desde enero de 2018 hasta septiembre de 2021, con las palabras clave: (personas trans) OR (transexualismo) OR (transexual) OR (identidad de género)) Y ((formación vocal) OR (voz) OR (voz calidad)). Resultados: se incluyeron 30 artículos científicos en el estudio. Conclusión: existe la necesidad de un seguimiento multidisciplinario y se ratifica la importancia de la logopedia, ya que el tratamiento hormonal y las cirugías laríngeas no incluyen todos los cambios de voz y habla deseados en las mujeres transgénero. Por lo tanto, el logopeda, además de buscar elevar f0, debe fomentar los gestos femeninos, los detalles de la voz y las delicadezas del habla. Se verificó la importancia de realizar cirugía laríngea asociada a logopedia para la modificación vocal de mujeres transgénero.

Palabras clave: Terapia vocal; Transgénero; Género y voz; Voz.

\section{Introdução}

As pessoas transgênero são um grupo de amplo espectro cuja identidade de gênero difere do sexo registrado no nascimento, sendo que muitos procuram tratamento médico para alinhar sua aparência física com sua identidade de gênero (Safer \& Tangpricha, 2019).

A abordagem terapêutica para os indivíduos transgênero envolve uma equipe multidisciplinar e considera as diferentes demandas necessárias para adequação ao gênero desejado, incluindo terapias psicoterápicas, hormonais, cirúrgicas e fonoaudiológicas (Gray \& Courey, 2019).

Nos homens transgênero, a terapia hormonal com testosterona proporciona aumento de massa das pregas vocais, agravamento da voz e, na maioria dos casos, não se faz necessária qualquer intervenção cirúrgica para adequação da frequência fundamental da voz (f0) (De Cuypere et al., 2006; Mcneill, Wilson, Clarck, \& Deakin, 2008). Entretanto, nos casos das mulheres transgênero, a terapia hormonal com estrogênio e outros hormônios femininos não interfere na f0 da voz. As mulheres transgênero têm f0 mais grave (na faixa masculina) do que as outras mulheres, além de diferentes características de ressonância e de fala, incluindo velocidade, entonação, loudness e expressão corporal. A f0 dessas mulheres deveria, no mínimo, estar em torno de $155 \mathrm{~Hz}$, levando-se em conta também outras características vocais, para que sejam percebidas como mulheres (Spiegel, 2006; Hancock, Colton, \& Douglas, 2014; Neumann \& Welzelm, 2004).

Considerando o reduzido número de estudos em português e a necessidade de elaboração das diretrizes para o tratamento vocal de mulheres transgênero, este estudo se propôs a fazer uma revisão não sistemática da literatura, sintetizando as abordagens empregadas, diretrizes terapêuticas e eventuais efeitos adversos com o intuito de contribuir com o clínico que trata essa população.

\section{Metodologia}

Trata-se de uma revisão bibliográfica por meio de pesquisa em artigos científicos nas bases de dados Pubmed e Science Direct sobre o tema disforia de gênero e voz, nos últimos 20 anos, sem restrições quanto ao tipo de estudo (revisões sistemáticas foram incluídas) e ao idioma de publicação. As palavras-chave utilizadas foram: ((Transgender Persons) OR (Transsexualism) OR (Transsexual) OR (Gender Identity)) AND ((voice training) OR (voice) OR (voice quality)). A pesquisa foi realizada pelos autores do estudo no período de janeiro de 2018 e agosto de 2021.

Como critérios de inclusão, considerou-se a literatura que continha a descrição de terapia, cirurgia ou técnicas vocais para a feminilização da voz, bem como sobre a satisfação vocal de mulheres transgênero; artigos científicos completos. Como critérios de exclusão: estudos com animais. Foram encontrados 326 artigos na busca e 31 foram incluídos no estudo. A revisão foi dividida em estudos sobre o tratamento cirúrgico, satisfação vocal e terapia fonoaudiológica para mulheres transgênero. 


\section{Resultados e Discussão}

\section{Tratamento Cirúrgico}

Quando o objetivo é aumentar a f0, o tratamento otorrinolaringológico com o recurso cirúrgico da aproximação cricotireoidea, também denominada de tireoplastia tipo IV é um dos mais citados na literatura (Spiegel, 2006; Neumann \& Welzelm, 2004; Borsel, Cuypere, Eynde, \& Bonte, 2008).

A cirurgia é realizada a partir da aproximação das cartilagens cricoide e tireoide no terço anterior laríngeo, ocasionando o estiramento permanente do músculo tiroaritenoideo. Isso promove a elevação da f0, tornando o pitch da voz mais agudo apesar de gerar limitação da variação melódica durante o discurso (Borsel, Cuypere, Eynde, \& Bonte, 2008). No entanto, autores referem que somente a realização do procedimento cirúrgico não é suficiente para a voz obter características femininas, sendo necessária a realização de terapia fonoaudiológica a fim de potencializá-las na nova voz (Spiegel, 2006; Borsel, Cuypere, Eynde, \& Bonte, 2008).

A tireoplastia tipo IV foi realizada em nove mulheres transgênero com aumento da média da f0 durante a leitura, situando-se na faixa de frequência feminina. O estudo concluiu que o procedimento de aproximação cricotireoidea é uma opção viável para elevar o pitch da voz de mulheres transgênero. No entanto, não é suficiente para criar uma voz que seja percebida como totalmente feminina, pois, além do pitch, outras características vocais necessitam ser modificadas (Borsel, Cuypere, Eynde, \& Bonte, 2008). Assim, a cirurgia pode ser indicada nos casos pós-terapia fonoaudiológica em que a f0 não tenha ultrapassado $155 \mathrm{~Hz}$ ou conforme a satisfação pessoal (Spiegel, 2006; Borsel, Cuypere, Eynde, \& Bonte, 2008).

Em um trabalho (Neumann \& Welzelm, 2004) com mulheres transgênero, constatou-se o aumento da f0 em 91\% (63) dos indivíduos que realizaram cirurgia de laringe (tireoplastia tipo IV) e tratamento fonoaudiológico posterior, com aumento de cinco a seis semitons na f0. Antes da cirurgia, nenhuma das características vocais das mulheres transgênero era feminina. No pós-operatório, $28 \%$ delas apresentava padrão mais feminino. Os autores recomendam a fonoterapia por um período mínimo de três meses, após a realização do procedimento cirúrgico, pois verificaram melhores resultados referentes à f0 em mulheres transgênero que realizaram fonoterapia em relação àquelas que apenas realizaram o procedimento cirúrgico.

Quatro estudos (Remacle, Matar, Morsomme, Veduickt, \& Lawson, 2011; Mastronikolis, Remacle, Biagini, Kiagiadaki, \& Lawson, 2013; Casado, Rodríguez-Parra, \& Adrián, 2017; Meister, Hagen, Shehata-Dieler, Kühn, Kraus, \& Kleinsasser, 2017) descreveram a cirurgia na laringe para a mudança vocal de mulheres transgênero por meio da técnica de Wendler, que consiste na ressecção do epitélio do terço anterior das pregas vocais e de sutura em forma de "V", promovendo o encurtamento e a consequente diminuição da massa vibratória. Todos encontraram aumento significativo da f0 média, porém ressaltou-se a importância da associação à terapia fonoaudiológica, a fim de melhorar o novo comportamento vocal, adaptar o sujeito à nova f0 da voz, ampliar a tessitura vocal e adequar certos marcadores femininos de fala (Mastronikolis, Remacle, Biagini, Kiagiadaki, \& Lawson, 2013; Casado, Rodríguez-Parra, \& Adrián, 2017).

Em uma revisão sistemática e meta análise (Schwarz et al., 2017a), verificou-se que não há evidências científicas suficientes sobre qual técnica cirúrgica é melhor para o tratamento vocal de mulheres transgênero. Outra revisão sistemática (Van Damme, Cosyns, Deman, Van den Eede, \& Van Borsel, 2017) evidenciou que a maioria dos pacientes estavam satisfeitos com os resultados da cirurgia, porém nenhum dos estudos utilizou grupo controle e processos de randomização. Além disso, há necessidade de investigações mais aprofundadas sobre os resultados em longo prazo. Neste caso, deve-se verificar a experiência do cirurgião em determinada técnica, bem como a preferência por uma técnica reversível ou irreversível.

Muitos indivíduos transgênero desconhecem ou não apresentam interesse em procurar um fonoaudiólogo para a realização de tratamento para a mudança da voz. Com isso, os ajustes adotados por essas pessoas podem, muitas vezes, resultar 
em hiperfunção do aparato fonador com geração de fadiga vocal e possíveis afecções laríngeas (Remacle, Matar, Morsomme, Veduickt, \& Lawson, 2011).

Diante do exposto, verifica-se na literatura que não há evidências de qual a melhor técnica para o procedimento cirúrgico de feminilização vocal (dentre as técnicas reversíveis e irreversíveis). Entretanto, a maioria dos autores concorda com a necessidade do tratamento fonoaudiológico como coadjuvante do tratamento médico cirúrgico. Cabe ao clínico conhecer as técnicas e avaliar com o cliente os riscos, desvantagens e benefícios de cada uma.

\section{Satisfação Vocal e Fonoterapia para Mulheres Transgênero}

Estudo (Palmer, Dietsch, \& Searl, 2012) com nove mulheres transgênero em tratamento hormonal, vivendo pelo menos $75 \%$ do tempo no papel feminino nos últimos seis meses, e com voz considerada feminina pelos interlocutores, mostrou sua crença em serem percebidas como mulheres em $85 \%$ das vezes em que falavam. Em relação à satisfação com a voz, numa escala de dez pontos, verificou-se média de 7,3 pontos. A respeito de qual método utilizaram para mudar a voz, duas pessoas referiram fonoterapia; quatro realizaram algum curso em casa com vídeos e livros; quatro praticavam em casa e trabalhavam com base nos ajustes que conseguiam realizar; e uma não realizou qualquer mudança de voz, pois sempre achou sua voz feminina. Observou-se constrição supraglótica em todas as mulheres transgênero; fechamento glótico incompleto com fenda posterior e diversas queixas vocais na maioria delas.

Uma pesquisa (Holmberg, Oates, \& Dacakis, 2016) utilizou fonetograma, medidas aerodinâmicas, questionário de autoavaliação e análise vocal perceptivoauditiva para avaliar as "vozes femininas" durante a leitura e a fala espontânea em loudness habitual e em loudness aumentada de 25 mulheres transgênero. Contrariamente a outras pesquisas, mostrou baixo índice de tipo de voz tensa e soprosa, não havendo indicações de comportamento vocal hiperfuncional. As análises vocais perceptivoauditivas foram realizadas por dois fonoaudiólogos experientes, além de 20 pessoas leigas que deveriam apenas classificar as vozes como femininas ou masculinas. Apesar do grupo analisado ser bastante heterogêneo, em diferentes estágios da terapia fonoaudiológica, os resultados da autoavaliação e da avaliação vocal perceptivoauditiva mostraram baixos índices de fadiga e disfunção vocal. Além disso, houve correlação positiva entre melhor percepção da voz e tempo de utilização da voz feminina, indicativo de que a melhora da voz pode ocorrer ao longo do tempo ou que as pessoas transgênero tendem a se habituar com a voz, deixando, assim, de ser um problema. Neste estudo, confirmou-se que uma f0 mais aguda com loudness fraca, sem uso de vocal fry, contribui com o sucesso da voz feminina nas mulheres transgênero.

Em outro estudo (Gelfer \& Schofield, 2000) com 15 mulheres transgênero), além de seis mulheres e três homens cisgênero (grupo de controle), as vozes dos participantes em leitura e vogais sustentadas foram julgadas por 20 estudantes de psicologia. Os resultados mostraram que os indivíduos percebidos como mulheres apresentavam maior média de f0 e maior alcance de frequências agudas do que os indivíduos percebidos como homens, havendo correlação positiva entre o maior alcance de frequências agudas e a percepção auditiva de feminilidade.

Mcneill, Wilson, Clarck e Deakin (2008) pesquisaram a relação entre a f0 da fala e a satisfação de mulheres transgênero após a fonoterapia verificou que a satisfação com a voz não estava relacionada somente à mudança de f0, mas que outros aspectos da voz como a entonação, a ressonância e a adoção do vocabulário feminino eram importantes no estabelecimento de uma voz aceitável.

Schwarz et al. (2018) verificaram que, no grupo de mulheres transgênero, o foco ressonante vertical estava mais alterado (mais nasal) e havia significativa rugosidade na voz. As vozes foram avaliadas de forma perceptivoauditiva como mais masculinas e a f0 era mais grave em relação ao grupo controle de mulheres cisgênero.

Gelfer e Tice (2013) investigaram em cinco mulheres transgênero a percepção da voz feminina e a f0 habitual da fala após oito sessões de fonoterapia, uma por semana, com duração de 60 minutos, sendo que as avaliações foram realizadas antes, 
imediatamente após e 15 meses depois da fonoterapia. O estudo fez uso de um grupo de controle composto por cinco mulheres e cinco homens cisgênero. Verificou-se que as mulheres transgênero foram percebidas como mulheres em 1,9\% das amostras no momento pré-terapia, 50,8\% após a terapia e 33,1\% 15 meses após. Em relação à f0, verificou-se que houve aumento imediatamente após a terapia e redução após 15 meses.

Dacakis, Davies, Douglas e Johnston (2013) realizaram a avaliação psicométrica do instrumento Transsexual Voice Questionnaire for Male-to-Female Transsexuals (TVQ-MtF), que tem como objetivo medir as percepções vocais das mulheres transgênero. Primeiramente, realizou se uma revisão do questionário Transgender Self-Evaluation Questionnaire (TSEQ) por dois fonoaudiólogos e duas mulheres transgênero. O TVQ-MtF foi elaborado, com um total de 30 itens, com uma escala Likert, para indicar a frequência dos acontecimentos (nunca ou raramente - 1, às vezes - 2, muitas vezes - 3 e normalmente ou sempre - 4). Após, 35 mulheres transgênero preencheram o questionário duas vezes num intervalo de quatro a seis semanas. Os autores verificaram que o TVQ-MtF forneceu relato confiável das sensações em relação à voz e ao impacto da voz sobre a vida da mulher transexual, demonstrando nível de consistência interna favorável e confiabilidade teste-reteste (Dacakis, Davies, Douglas, \& Johnston, 2013; Dacakis G, Oates J, \& Douglas, 2017).

O mesmo questionário validado para o português (Santos, Aguiar, Baeck, \& Van Borsel, 2015) foi aplicado em 47 mulheres transgênero e verificou-se que a maioria das participantes apresentou escores baixos no TVQMtF. As diferenças significativas no escore total foram observadas de acordo com a idade (escores mais elevados foram observados entre aquelas com menos de 30 anos e $\geq 40$ anos) e em relação às avaliações visuoperceptivas do rosto (maiores escores foram observados no indivíduo avaliado com face mais masculina) (Schwarz et al., 2017b).

Hancock, Colton e Douglas (2014) analisaram a influência do padrão de entonação para a percepção de gênero em mulheres e homens transgênero. Para a realização da análise vocal perceptivoauditiva, participaram 14 indivíduos com audição normal autorrelatada, aos quais foram apresentadas 54 vozes, sendo 12 masculinas, 12 femininas, seis homens transgênero, 14 mulheres transgênero e dez repetidas. Os juízes analisaram as vozes e marcaram em uma escala visual na qual a esquerda correspondia à voz com características mais masculinas e a direita à voz mais feminina. Os juízes conseguiram diferir 100\% das vozes femininas e masculinas, sendo que cinco das vozes dos homens transgênero foram identificadas como masculinas; entretanto, das vozes das 14 mulheres transgênero, apenas quatro foram identificadas como voz feminina. As mulheres transgênero que não passaram como voz feminina não usavam adequadamente os aspectos de entonação dificultando o julgamento da voz. Assim, enfatizou-se a necessidade desse aspecto também ser objetivo da terapia fonoaudiológica.

Em um relato de caso sobre fonoterapia com uma adolescente mulher transgênero de 15 anos, utilizaram-se técnicas de feedback auditivo, por meio de gravações de vozes, e posterior utilização de programas computacionais que identificavam a f0 e o nível de pressão sonora. O tratamento abordou a higiene vocal, o suporte respiratório, a f0, a entoação, a ressonância e a qualidade vocal. Os resultados positivos, tanto perceptuais como de autorrelato da cliente confirmaram a eficácia do tratamento (Hancock \& Helenius, 2012).

A abordagem de tratamento sintomática (Gelfer \& Tice, 2013) com o uso de Exercícios de Função Vocal, foi verificada em um estudo com três mulheres transgênero que buscavam a modificação da voz. As vozes foram comparadas a dois grupos de controle, respectivamente, de três homens cisgênero e de três mulheres cisgênero. As mulheres transgênero receberam 12 sessões de terapia durante seis semanas. Nas medidas vocais acústicas, as mulheres transgênero apresentaram resultados semelhantes ao grupo de controle de homens cisgênero antes do tratamento e ao grupo controle de mulheres cisgênero após o tratamento, destacando-se o aumento da f0. Na avaliação vocal perceptivoauditiva, realizada por 27 juízes não fonoaudiólogos, no pré-tratamento todas as vozes foram consideradas masculinas e no pós-tratamento, embora a maioria das vozes ainda tenha sido considerada masculina, houve melhora, com 7,4\% das identificações como femininas. 
Neumann e Welzelm (2004) verificaram a importância da realização de cirurgia de laringe combinada à terapia fonoaudiológica em mulheres transgênero. Durante a terapia de voz, os aspectos trabalhados foram: a prosódia, o vocabulário e a articulação mais suave. Após um ano, 58\% das mulheres transgênero que receberam fonoterapia pós-cirurgia mostraram f0 mais alta e mais estável do que aquelas que não foram submetidas à terapia.

Não há consenso na literatura no que se refere à frequência semanal e tempo total da fonoterapia para os casos de pessoas transgênero. O Conselho Federal de Fonoaudiologia Brasileiro (2013) lançou o guia balizador de tempo de tratamento em Fonoaudiologia e, na área de voz, o número de sessões varia de uma a três vezes por semana, com duração de 30 a 45 minutos, sem referência específica a casos de pessoas transgênero. Não foi encontrada correlação entre o número de sessões de fonoterapia e o alcance de uma f0 mais aguda, sugerindo que esse aspecto está mais relacionado a fatores individuais e que a fonoterapia deve ser planejada de forma exclusiva para cada paciente levando-se em conta as características de cada um.

A terapia de voz de ressonância oral foi aplicada em dez mulheres transgênero que realizaram cinco sessões de terapia visando ao estiramento dos lábios e ao posicionamento da língua mais anterior (Carew, Dacakis, \& Oates, 2007). Os resultados da análise acústica pré e pós-terapia mostraram que os valores de frequência de formantes (F1, F2 e F3, das vogais /a/, /i/ e /ひ/) e da f0 aumentaram pós-terapia. Os valores de F3, principalmente, aumentaram significativamente pós-tratamento. Na análise perceptivoauditiva, a maioria das participantes foi percebida como mais feminina após o tratamento. As autoavaliações vocais das participantes pré e pós-tratamento evidenciaram vozes soando mais femininas e maior satisfação vocal após o tratamento. O estudo fornece evidências preliminares que sugerem que a terapia de ressonância oral pode ser eficaz no aumento da feminilidade da voz em mulheres transgênero.

Hollien, Bahr e Harnsberger (2013) também identificaram por meio do biofeedback visual-acústico que valores mais altos de F2 foram associados a aumento na feminilidade percebida da fala e, em conjunto com a f0, contribuem para a percepção de gênero. Os autores sugerem que o biofeedback pode ser uma ferramenta útil na terapia de modificação de voz para mulheres transgênero.

Ainda não há padronização sobre qual o tipo de terapia fonoaudiológica é a mais efetiva para a modificação vocal de mulheres transgênero. Os resultados de Gelfer e Van Dong (2013) sugerem que o tratamento sintomático da voz com Exercícios de Função Vocal tem um papel importante na modificação da voz, embora abordagens fisiológicas também possam ser indicadas de maneira complementar.

Revisão sistemática e meta-análise (Leung, Oates, \& Chan, 2018) abordou os aspectos da comunicação verbal e percepções do ouvinte sobre o sexo do falante para fornecer aos clínicos orientação na seleção de técnicas e objetivos do treinamento fonoaudiológico no atendimento de pessoas transgênero. Neste estudo, os autores verificaram que a f0 de fala contribuiu em 41,6\% na variância da percepção de gênero. Os cinco principais componentes que contribuem para as percepções do ouvinte sobre o sexo do falante são: pitch, ressonância, loudness, articulação e entonação. Mesmo que alguns autores tenham sugerido que sintaxe, léxico, comunicação não-verbal e comunicação pragmática possam ser usados no treinamento de comunicação das pessoas transgênero, não há evidências da efetividade destas características na identificação do sexo do falante. As frequências dos quatro primeiros formantes de vogais (F1, F2, F3 e F4) demonstraram empiricamente contribuir para a percepção do gênero. $\mathrm{O}$ treinamento de voz para atingir altas frequências nos primeiros quatro formantes de vogais provavelmente contribui para a percepção de um falante feminino. Devido às limitações das medidas perceptivoauditivas e acústicas da voz para avaliar se a soprosidade contribui para a percepção do ouvinte sobre o gênero, este recurso não deve ser usado no treinamento.

Gray e Courey (2019) em uma revisão de literatura sobre o tratamento vocal de pessoas transgênero concluiu que mudanças comportamentais no estilo de comunicação e voz são os objetivos principais da terapia e que existem muitas variações de técnicas e estratégias para obtenção e manutenção de uma voz feminina. Essas variações acontecem 
principalmente em razão da complexidade e individualidade humana, expectativas vocais, bem como a falta de tratamento padronizado. Além disso, a fonocirurgia foi citada como um complemento necessário para o auxiliar o alcance de uma voz ideal.

Revisão sistemática e meta-análise (Nolan et al., 2019) sobre tratamento vocal com 20 artigos incluídos verificou que a satisfação do paciente pós-intervenção foi aproximadamente $80 \%$ a $85 \%$ para terapia vocal, cirurgia endoscópica de encurtamento e aproximação cricotireoidiana. As complicações mais relatadas para as técnicas cirúrgicas foram diminuição do tempo máximo de fonação e da loudness. Em relação aos ganhos da f0 após tratamento, evidenciou-se $31 \mathrm{~Hz}$ somente com a terapia de voz, $26 \mathrm{~Hz}$ com glotoplastia de redução a laser, $39 \mathrm{~Hz}$ com aproximação cricotireoidiana e $72 \mathrm{~Hz}$ com encurtamento endoscópico. Os autores também concluíram que a literatura apoia tanto a terapia vocal quanto fonocirurgia.

Outro aspecto importante refere-se ao tratamento precoce. No caso de meninos que realizam o tratamento de supressão da puberdade com o uso de hormônios análogos das gonadotrofinas, tratamento ainda em caráter de pesquisa no Brasil, não ocorre o agravamento da voz esperado na puberdade e, desta forma, a f0 tende a permanecer na faixa feminina e nem sempre será necessário o tratamento vocal (Schneider et al., 2019).

\section{Conclusão}

$\mathrm{O}$ tratamento para indivíduos transgênero ainda gera muitos questionamentos em decorrência do limitado número de estudos de evidência científica, entretanto, por serem casos complexos que envolvem aspectos biológicos, sociais e emocionais, afirma-se a necessidade do acompanhamento multiprofissional.

Por mais que os aspectos físicos e emocionais estejam de acordo com o novo gênero, ratifica-se a importância da fonoterapia, uma vez que o tratamento hormonal e as cirurgias laríngeas sozinhas não contemplam todas as modificações desejadas na voz e fala das pessoas transgênero. O fonoaudiólogo tem um papel fundamental no atendimento da população transgênero, já que a terapia vocal é a primeira opção de tratamento para a feminilização vocal, na maioria dos casos, coadjuvante ou não ao tratamento cirúrgico.

Quanto às possibilidades fonoterapêuticas, a literatura aponta o uso de algumas estratégias como elevação e modulação de f0, treinamento de voz para atingir altas frequências nos primeiros quatro formantes de vogais, redução e variação de loudness, melhora da articulação e entonação, técnicas de feedback auditivo, Terapia de Voz de Ressonância Oral e Exercícios de Função Vocal.

Os fonoaudiólogos que trabalham com indivíduos transgênero podem usar os resultados dessa revisão para estabelecer metas de terapia. No entanto, mais pesquisas são necessárias para corrigir o risco significativo de viés.

\section{Agradecimentos}

O presente trabalho foi realizado com apoio da Coordenação de Aperfeiçoamento de Pessoal de Nível Superior Brasil (CAPES) - Código de Financiamento 001 e Conselho Nacional de Desenvolvimento Científico e Tecnológico (CNPQ) (Grant 301326/2017-7) e Fundação de Amparo à Pesquisa do Estado do Rio Grande do Sul (grant INCT/FAPERGS: 17/25510000519-8).

\section{Referências}

Borsel, J. V., Cuypere De, G., Eynde, E. V., \& Bonte, K. (2008). Feminine after cricothyroid approximation? Journal of Voice, 22(3), 379-84. 10.1016/j.jvoice.2006.11.001.

Borsel, J. V., Pot, K., \& Cuypere, G. (2009). Voice and physical appearance in female-to-male transsexuals. Journal of Voice, 23(4), 494-7. 10.1016/j.jvoice.2007.10.018. 
Borsel, J. V., \& Baeck, H. (2014). The voice in transsexuals. Revista de Logopedia, Foniatría y Audiología, 34(1), 40-48. https://doi.org/10.1016/j.rlfa.2013.04.007

Casado, J. C., Rodríguez-Parra, M. J., \& Adrián, J. A. (2017). Voice feminization in male-to-female transgendered clients after Wendler's glottoplasty with vs. without voice therapy support. European Archives of Oto-rhino-laryngologic, 274(4), 2049-2058. 10.1007/s00405-0164420-8

Carew, L., Dacakis, G., \& Oates, J. (2007). The effectiveness of oral resonance therapy on the perception of femininity of voice in male-to-female transsexuals. Journal of Voice, 21(5), 591-603. 10.1016/j.jvoice.2006.05.005

Dacakis, G., Davies, S., Douglas, J. M., \& Johnston, J. R. (2013). Development and preliminary evaluation of transsexual voice questionnaire for male-tofemale transsexuals. Journal of Voice, 27(3), 312-20. 10.1016/j.jvoice.2012.11.005

Dacakis, G., Oates, J., \& Douglas, J. (2017). Associations between the Transsexual Voice Questionnaire (TVQMtF ) and self-report of voice femininity and acoustic voice measures. International Journal of Language \& Communication Disorder, 52(6), 831-838. 10.1111/1460-6984.12319

De Cuypere, G., Elaut E., Heylens G.,Van Maele, G., Selavaggi G, Tsjoen, G., \& Monstrey, S. (2006). Long term follow-up: psychosocial outcome of Belgian transsexuals after sex reassignment surgery. Sexologies, 15(2), 126-33. https://doi.org/10.1016/j.sexol.2006.04.002

Conselho Federal de Fonoaudiologia (CFFa). (2013). Guia de orientação para fonoaudiólogos: balizador de tempo de tratamento em fonoaudiologia. Organização: Grupo de Trabalho instituído pela comissão de saúde do CFFa, Academia Brasileira de Audiologia e Sociedade Brasileira de Fonoaudiologia. http://www.fonoaudiologia.org.br/publicacoes/BALIZADOR\%20DE\%20TEMPO.pdf

Gelfer, M. P, \& Schofield, K. J. (2000). Comparison of acoustic and perceptual measures of voice in male-to-female transsexuals perceived as female versus those perceived as male. Journal of Voice, 14(1), 22-33. 10.1016/s0892-1997(00)80092

Gelfer, M. P., \& Dong, B. R. V. (2013). A preliminary study on the use of Vocal Function Exercises to improve voice in male-to-female transgender clients. Journal of Voice, 27(3), 321-34. 10.1016/j.jvoice.2012.07.008

Gelfer, M. P., \& Tice, R. M. (2013). Perceptual and acoustic outcomes of voice therapy for male-to-female transgender individuals immediately after therapy and 15 months later. Journal of Voice, 27(3), 335-47. 10.1016/j.jvoice.2012.07.009

Gray, M. L., \& Courey, M. S. (2019). Transgender Voice and Communication. Otolaryngologic Clinics of North America, 52(4), 713-722. 10.1016/j.otc.2019.03.007

Hancock, A. B., \& Helenius, L. (2012). Adolescente male-to-female transgender voice and communication therapy. Journal of Communication Disorder, 45(5), 313-24. 10.1016/j.jcomdis.2012.06.008

Hancock, A. B., Colton L., \& Douglas, F. (2014) Intonation and Gender Perception: Applications for Transgender Speakers. Journal of Voice, 28(2), 203-9. https://doi.org/10.1016/j.jvoice.2013.08.009

Holmberg, E. B., Oates, J., \& Dacakis, G. (2010). Phonetograms, aerodynamic measurements, self evaluations, and auditory perceptual ratings of male-tofemale transsexual. Journal of Voice, 24(5), 511-22. 10.1016/j.jvoice.2009.02.002

Hollien, H., Bahr, R. H., \& Harnsberger, J. D. (2014). Issues in Forensic Voice. Journal of Voice, 28(2), 170-84. 10.1016/j.jvoice.2013.06.011

Leung, Y., Oates, J., \& Chan, S. P. (2018). Voice, Articulation, and Prosody Contribute to Listener Perceptions of Speaker Gender: A Systematic Review and Meta-Analysis. Journal of Speech, Language and Hearing Research, 61(2), 266-297. 10.1044/2017_JSLHR-S-17-0067

Mastronikolis, M. S., Remacle, M., Biagini, M., Kiagiadaki, D., \& Lawson, G. (2013). Glottoplasty: An effective pitch raising surgery in male-to-female transsexuals. Journal of Voice, 27 (4), 516-22. 10.1016/j.jvoice.2013.04.004

Mcneill, E. J. M., Wilson, J. A., Clarck, S., \& Deakin, J (2008). Perception of voice in the transgender client. Journal of Voice, 22(6), 727-33. https://doi.org/10.1016/j.jvoice.2006.12.010

Meister, J., Hagen, R., Shehata-Dieler, W., Kühn, H., Kraus, F., \& Kleinsasser, N. (2017) Pitch Elevation in Male-to-female Transgender Persons-the Würzburg Approach. Journal of Voice, 31(2), 244.e7-244.e15. 10.101 6/j.jvoice.2016.07.018

Neumann, K., \& Welzelm, C. (2004). The importance of the voice in male-to-female transsexualism. Journal of Voice, 18(1), 153-67. 10.1016/S08921997(03)00084-5

Nolan, I. T., Morrison, D. S., Arowojolu, O., Crowe, C. S., Massie, J. P., Adler, R. K., \& Francis, D. O. (2019). The Role of Voice Therapy and Phonosurgery in Transgender Vocal Feminization. The Journal of Craniofacial Surgery, 30(5),1368-1375. 10.1097/SCS.0000000000005132

Palmer, D., Dietsch, A., \& Searl, J. (2012). Endoscopic and stroboscopic presentation of the larynx in male-to-female transsexual persons. Journal of Voice, 26(1),117-26. 10.1016/j.jvoice.2010.10.014

Remacle, M., Matar, N., Morsomme, D., Veduickt, I., \& Lawson, G. (2011). Glottoplasty for male-to female transsexualism: voice results. Journal of Voice, 25(1), 120-23. 10.1016/j.jvoice.2009.07.004

Safer, J. D., Tangpricha, V. (2019). Care of the Transgender Patient. Annals of Internal Medicine, 171(1), ITC1-ITC16. 10.7326/AITC201907020

Santos, H. H., Aguiar, A. G., Baeck, H. E., \& Van Borsel, J. (2015). Translation and preliminary evaluation of the Brazilian Portuguese version of the Transgender Voice Questionnaire for male-to-female transsexuals. Codas, 27(1), 89-96. 10.1590/2317-1782/20152014093 
Research, Society and Development, v. 10, n. 14, e247101421651, 2021

(CC BY 4.0) | ISSN 2525-3409 | DOI: http://dx.doi.org/10.33448/rsd-v10i14.21651

Schneider, M. A., Spritzer, P. M., Soll, B. M. B., Fontanari, A. M. V., Carneiro, M., Tovar-Moll, F., \& Lobato, M. I. R. (2017). Brain Maturation, Cognition and Voice Pattern in a Gender Dysphoria Case under Pubertal Suppression. Front Hum Neurosci. 14(11), 528. 10.3389/fnhum.2017.00528

Schwarz, K., Fontanari, A. M. V., Schneider, M. A., Soll, B. M. B., da Silva D. C., Spritzer, P. M., \& Lobato M. I. R. (2017a). Laryngeal surgical treatment in transgender women: A systematic review and meta-analysis. Laryngoscope. 127(11), 2596-2603. 10.1002/lary.26692

Schwarz, K., Fontanari, A. M. V., Mueller, A., Costa, A. B., Soll, B. M. B., da Silva, D. C., \& Lobato, M. I. R. (2017b). Transsexual Voice Questionnaire for Male-to-female Brazilian Transsexual People. Journal of Voice, 31(1), 120.e15-120.e20. 10.1016/j.jvoice.2016.02.012

Schwarz, K., Fontanari, A. M. V., Costa, A. B., Soll, B. M. B., da Silva, D. C., Villas-Bôas, A. P. S., \& Lobato, M. I. R. (2018). Perceptual-Auditory and Acoustical Analysis of the Voices of Transgender Women. Journal of Voice, 32(5), 602-608. 10.1016/j.jvoice.2017.07.003

Spiegel, J. H. (2006) Phonosurgery for pitch alteration: feminization and masculinization of the voice. Otolaryngologic Clinics of North America, 39(1): 7786. 10.1016/j.otc.2005.10.011

Van Damme, S., Cosyns, M., Deman, S., Van den Eede, Z., \& Van Borsel, J. (2017). The Effectiveness of Pitch-raising Surgery in Male-to-Female Transsexuals: A Systematic Review. Journal of Voice, 31(2), 244.e1-244.e5. 10.1016/j.jvoice.2016.04.002 